\title{
RGB Analysis of Wedge Angles Around a Perforation in Silicon
}

\author{
Daugherty, Jamie ${ }^{1}$ and Fraundorf, P. ${ }^{2}$ \\ 1. University of Missouri- Saint Louis, Department of Physics, Saint Louis MO, USA \\ 2. University of Missouri- StL, Center for NanoScience \& Dept. of Physics, Saint Louis MO, USA
}

Measuring the wedge angle and specimen thickness of $3 \mathrm{~mm}$ perforated TEM samples can be difficult. Semiconductor silicon exhibits useful color changes in transmitted light, from opaque to red to yellow to white, as its thickness decreases below a few microns. This paper will describe progress with algorithms for mapping specimen thickness and hence wedge angle at selected regions in such specimens.

To begin the process, a perforated specimen is lighted from beneath, allowing light to shine through the hole and thinner portions of the sample. The sample should be placed on a small puncture in dark paper, allowing only light from directly beneath the specimen to come through. Other extraneous light from the room should be mitigated to prevent reflected light from around and above the specimen from contaminating the data. Once the effect from external light is negligible and the only transmitted light is coming from beneath the specimen, digital images from a light microscope can be recorded.

The images may now be used to analyze the wedge angle by finding the thickness of a sample in two places, and analyzing that data as the "rise" and "run" of a triangle to obtain the degree of the angle. Open the file in a program such as Microsoft Paint, Image J or Photoshop and locate the area under surveillance. Using the pixel count given by the program, choose a pixel in the "hole of your specimen, recording that pixel's $x$ and y coordinates as well as its RGB numbers. Next, choose a corresponding pixel outside the hole and record its data. Using the $\mathrm{x}$ and $\mathrm{y}$ coordinates obtained from both pixels, calculate the difference on both the $\mathrm{x}$ and $\mathrm{y}$ axis, and use those to get the length of your line: $\sqrt{ }\left[\left(\mathrm{X}_{2}-\mathrm{X}_{1}\right)^{2}\right.$ $\left.+\left(\mathrm{Y}_{2}-\mathrm{Y}_{1}\right)^{2}\right]$. This length will be considered the "run" for calculating the angle. The distance in pixels can be changed into units of length measurement using the image field width, or a length standard imaged under the same conditions.

To find the "rise," use the RGB numbers obtained from each pixel and insert them into the equation $\left[\left(G / G_{0}\right) /\left(R / R_{0}\right)\right]$ where $G$ is the green number on the specimen, $G_{0}$ is the green measurement in the hole, $\mathrm{R}$ is the red number on the specimen, and $\mathrm{R}_{0}$ is the red measurement in the hole. The result obtained will be defined as $(\mathrm{G} / \mathrm{R})$, the green to red attenuation. The thickness of the specimen can be acquired from the function $(\mathrm{G} / \mathrm{R}) \approx 1-0.217 \mathrm{t}+0.0165 \mathrm{t}^{2}$, where $\mathrm{t}$ is the thickness. This function was derived from the color of silicon transmission chart [1]. The thickness versus attenuation is presented in figure 1. For better accuracy, several lines were chosen to measure the variables and an average of the group was used to calculate all data.

Once the rise and run are found, the equation $\tan \theta=$ rise/run can be used to compute the wedge angle of the specimen. Wedge angles found for specimen 1 and specimen 2 were $13.1^{\circ}$ and $15.7^{\circ}$, respectively. The average wedge angle calculated by other methods for specimen 1 was $13.6^{\circ}$, while the calculation for specimen 2 was $17.0^{\circ}$. This shows an average percent error for the two specimens at $5.7 \%$. Since the area where the wedge angle was measured was approximated in all cases, this error is relatively small. 
Through examination of the results from this method and the comparison of outcomes from other methods, it is clear that this method of analyzing wedge angles in perforated silicon specimens is a valid technique. This approach to calculating wedge angles is both fast and reliable, and can be used as an effective mode of acquiring results [3].

\section{References:}

[1] J.P. McCaffrey, B.T. Sullivan, J.W. Frase, D.L. Callahan (1996) Use of transmitted color to calibrate the thickness of silicon samples. Micron, 27, 407-411

[2] P. Hirsch, A. Howie, R. Nicolson, D.W. Pashley and M.j. Whelan (1965/1977) Electron microscopy of thin crystals (Butterworths/Krieger, London/Malabar FL) ISBN 0-88275-376-2 [3] The authors acknowledge funding from the Missouri Space Grant Consortium. David Osborn, Ashlynn Conner, and Stephen Ordway are thanked for their assistance, and Stephen Ordway is thanked for his thickness fringe data on the $2^{\text {nd }}$ specimen.

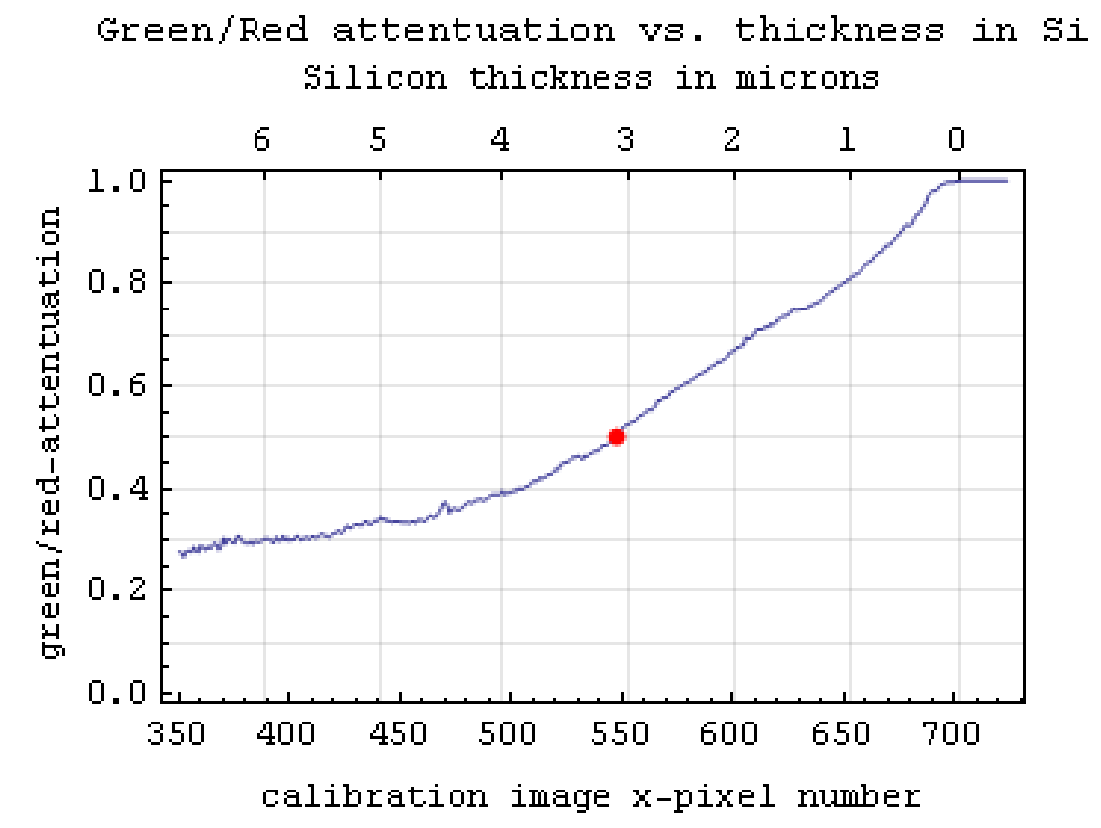

Figure 1. Green/Red Thickness approximation chart
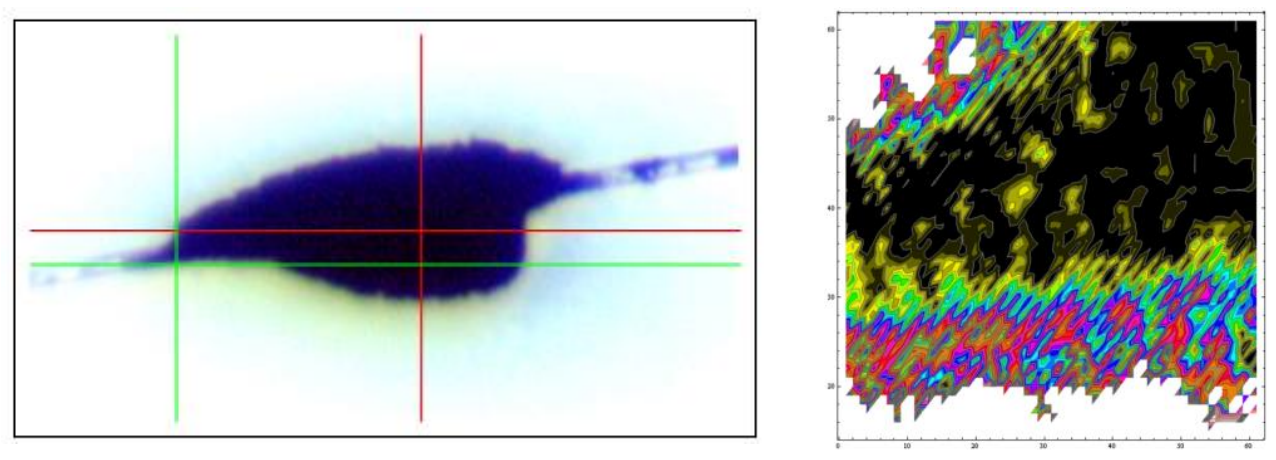

Figure 2. Specimen 2 silicon sample image detailing hole and edge locations and color contour map showing specimen thickness around the hole 